\title{
Elevated Temperature Observation of Selective Oxidation Catalyst M1 Along [001]
}

\author{
D. A. Blom,* L. F. Allard** and W. C. Bigelow***
}

* Electron Microscopy Center, University of South Carolina, 715 Sumter St., CLS 001, Columbia, SC 29208

**Materials Science and Technology Division, Oak Ridge National Laboratory, Oak Ridge, TN 37831-6064

*** Dept. of Materials Science and Engineering, University of Michigan, Ann Arbor, MI 48104

Selective oxidation catalysts are used extensively in the production of intermediate products and final consumer goods from a variety of feedstocks. $M 1$ is a Mo-V-Nb-Te-O phase with a large orthorhombic unit cell which is made up of a series of pentagonal, hexagonal and heptagonal rings of metal-oxygen octrahedra [1-2]. It is one phase of the most promising candidate material for the partial ammoxidation reaction of propane and ammonia to produce acrylonitrile (ACN) [3] and is the phase which can directly convert propane to ACN with high activity. Unlike many catalysts which consist of an active species on a support, the Mo-V-Nb-Te-O crystal is directly responsible for the catalytic activity The current process for making ACN involves reacting propene and ammonia over a multi-phase multicomponent bismuth molybdate catalyst [4-5]. Changing from a propene feedstock to propane is projected to yield a cost savings of $\$ 400$ million per year due to the cost difference of the feedstocks and the large quantity of ACN produced each year [3].

The structural details of the $M 1$ phase have been the focus of much research over the past 20 years. A number of synthesis methods have been reported with a variety of compositions and range of activity and selectivity [4-6]. Aberration-corrected HAADF STEM imaging has been recently used to characterize $M 1$ and some related phases at room temperature [7-10]. Because the $M 1$ unit cell is only a single metal-oxygen octahedron thick along the $c$-axis, HAADF STEM of the $<001>$ orientation of the $M 1$ phase shows varying signal intensity from the 11 framework cation sites which are known to exhibit varying amounts of $\mathrm{V}$ substitution for Mo [1-2,7]. Recently, Korovchenko et al. [5] have reported the phase transformation of $M 1$ to the pseudo-hexagonal $M 2$ phase for certain compositions at $873 \mathrm{~K}$. Pyrz et al. have reported the intergrowth of $M 1$ with a $\mathrm{Mo}_{5} \mathrm{O}_{14}$-type phase [10]. Optimal activity and selectivity of the $M 1$ phase for production of ACN occurs at 703K [6]. It is not clear that the details of the structure will remain constant at the operating conditions, particularly in light of the above recent reports. We will report on the changes in the structure of $M I$ crystallites observed via aberration-corrected HAADF STEM imaging in a $<001>$ orientation as a function of temperature.

Needles of $M 1$ with a diameter or 100 to $200 \mu \mathrm{m}$ and lengths of several hundred microns with a $c$ axis growth direction are typically reported [11]. A STEM shadow image showing several MI crystallites supported on a C-flat ${ }^{\mathrm{TM}}$ film on a Protochips Aduro MEMs-heating device is seen in Fig. 1a. The hole in the ceramic heating membrane is $5 \mu \mathrm{m}$ in diameter. The highlighted crystallite is seen in the HAADF STEM image of Fig. 1b after double-axis tilting to orient to a [001] zone axis condition. The area highlighted in Fig. $1 \mathrm{~b}$ is seen at higher magnification in Fig. 1c. The large pores in the structure are the heptagonal ring which is partially filled with $\mathrm{Te}-\mathrm{O}$ chains along [001]. The 
pentagonal rings are clearly visible in the image with the central atomic column containing the $\mathrm{Nb}$ in the specimen. The hexagonal rings are substantially filled with $\mathrm{Te}-\mathrm{O}$ in Fig. 1c.

The advantages of observation along a $<001>$ direction for $M 1$ are obvious from Fig. 1c. The cation sites do not overlap in this projection and consequently the composition of the various cation sites can be inferred from the HAADF STEM images [7]. In order to study the structure of $M 1$ at temperatures relevant for catalytic activity with electron microscopy, we have used a newly fabricated double-tilting specimen rod designed to operate with the Protochips Aduro heating devices [11]. This holder allows even small particles to be aligned appropriately, and most importantly, for the alignment to be corrected after heating to accommodate movement of the chosen particle due to the heating effects. Figure 1c was taken with a sample of $M 1$ at room temperature and illustrates both the double-tilting capability and the stability of the rod for atomic-level imaging [12].

\section{References}

[1] P. DeSanto Jr. et al., Z. Kristallogr. 219 (2004) 152.

[2] X. Li et al., Top. Catal. in press (2011).

[3] R. K. Grasselli, Top. Catal. 21 (2002) 79.

[4] R. K. Grasselli et al. Top. Catal. 48 (2006) 7.

[5] P. Korovchenko et al. Top. Catal. 50 (2008) 43.

[6] N. Watanabe and W. Ueda, Ind. and Eng. Chem. Res. 45 (2006) 607.

[7] W. D. Pyrz et al. Angew. Chem. Int. Ed. 47 (2008) 2788.

[8] D. A. Blom et al. J. Electron Microsc. 58 (2009) 193.

[9] W. D. Pyrz et al. Chem. Mater. 22 (2010) 2033.

[10] W. D. Pyrz et al. Proc.Natl. Acad. Sci. USA 107 (2010) 6152.

[11] L. F. Allard et al. this proceedings.

[12] This research at the Oak Ridge National Laboratory's High Temperature Materials Laboratory was sponsored by the U. S. Department of Energy, Office of Energy Efficiency and Renewable Energy, Vehicle Technologies Program.

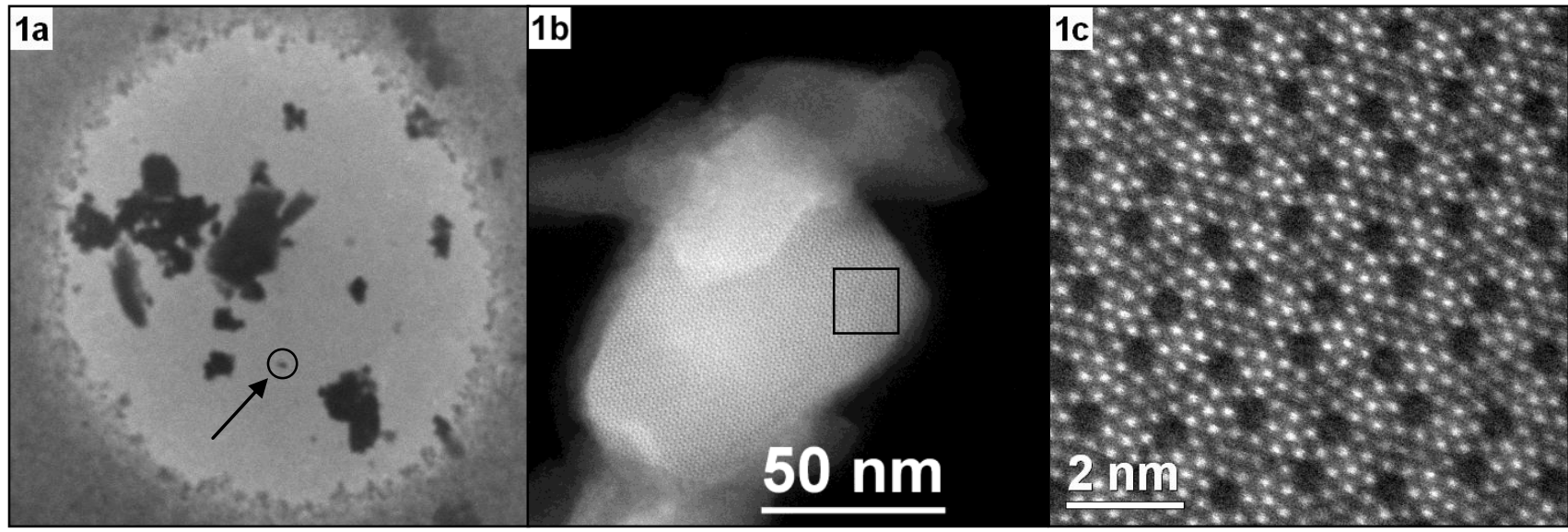

FIG. 1. a) STEM shadow image of $M 1$ specimen on Protochips Aduro heating device (hole $\sim 5 \mu \mathrm{m}$ ). b) HAADF STEM of crystallite highlighted in Fig. 1a after alignment along the [001] zone axis. c) High-resolution HAADF STEM of orthorhombic $M 1$ phase at room temperature. Images were acquired on the JEOL 2200FS aberration-corrected STEM at ORNL. 